\title{
SAÚdE MENTAL NA ATENÇÃO BÁSICA COMO PROCESSO HISTÓRICO DE EVOLUÇÃO DA PSIQUIATRIA COMUNITÁRIA
}

\author{
Mental Health in the Basic Attention as Historical Process \\ of Community Psychiatry Evolution \\ Salud Mental en la Atención Básica como Porceso Histórico de \\ Evolución de la Psiquiatría Comunitaria
}

Amanda Márcia dos Santos Reinaldo ${ }^{1}$

\section{Resumo}

0 artigo se propõe a analisar aspectos teóricos sobre a saúde mental na atenção básica e sua interface com a psiquiatria comunitária a partir de uma reflexão sobre a produção científica relacionada ao tema. Para tanto, foi realizada uma revisão bibliográfica no período de maio a julho de 2005, quando foram consultadas as bases de dados (Biblioteca Virtual em Saúde, Bireme, Scielo, Lilacs) e fontes primárias que versam sobre o tema, utilizando as palavras-chave: saúde mental, atenção primária, psiquiatria, comunidade, atenção básica. Foram encontradas 142 ocorrências e, destas, utilizadas 20 de acordo com o objetivo do estudo. A análise do material compilado subsidia a discussão de que efetivamente há uma interface entre a saúde mental na atenção básica e a psiquiatria comunitária que se reflete na atual política de saúde mental.

Palavras-chave: Saúde Mental. Atenção Primária à Saúde. Psiquiatria Comunitária.

\begin{abstract}
This article aims to analyze theoretical aspects of mental health in basic care and its interface with community psychiatry, based on a reflection about scientific production related to the theme. Therefore, a bibliographic review was carried out between May and July 2005, consulting databases (Virtual Health Library, Bireme, Scielo, Lilacs) and primary sources about the theme, using the keywords: mental health, primary care, psychiatry, community, basic care. In total, 142 occurrences were found, 20 of which were used according to the study objective. The analysis of the compiled material supports the discussion about the actual existence of an interface between mental health in basic care and community psychiatry, which is reflected in the current mental health policy.
\end{abstract}

Keywords:

Mental Health. Primary Health Care. Community Psychiatry.

\section{Resumen}

La finalidad del artículo es analizar aspectos teóricos sobre la salud mental en la atención básicay su interfaz con la psiquiatría comunitaria a partir de una reflexión sobre la producción científica relacionada al tema. Para tanto fue realizada una revisión bibliográfica en el período de mayo a julio de 2005 , donde fueron consultadas las bases de datos (Biblioteca Virtual en Salud, Bireme, Scielo, Lilacs) y fuentes primarias sobre el tema, utilizando las palabras-clave: salud mental, atención primaria, psiquiatría, comunidad, atención básica. Fueron encontradas 142 ocurrencias y, de estas, utilizadas 20 de acuerdo con el objetivo del estudio. El análisis del material compilado subsidia la discusión de que efectivamente existe una interfaz entre la salud mental en la atención básica y la psiquiatría comunitaria que se refleja en la actual política de salud mental. 


\section{INTRODUÇÃO}

0 tratamento asilar caracterizado pelo isolamento da comunidade e desrespeito aos direitos humanos é incompatível com a política de atenção em saúde mental que prevê a prevenção da doença, a promoção da saúde, a descentralização, o envolvimento da população e um enfoque comunitário em relação às suas ações.

Analisando os projetos da Medicina Integral, Medicina Preventiva e Medicina Comunitária, observamos que esses movimentos foram marcados por uma tentativa de integração da dimensão social, presente na produção das enfermidades que vinham sendo excluídas do ato médico. Já nas décadas de 1940 e 1950, essa preocupação em globalizar a prática individualizada pelas especialidades colabora para a idéia do indivíduo como "totalidade biopsicossocial irredutível a um conjunto de estruturas e funções orgânicas" 1:79 .

É a partir desse momento que o sujeito é visto como um ser global, portanto, necessitando de atenção multiprofissional. A Medicina Comunitária, agregando conhecimentos da Medicina Integral e da Preventiva, passa a observar o processo saúdedoença sob uma dimensão ecológica e psicossocial ${ }^{1,2}$.

0 termo psicossocial "vai ser transladado para o campo das práticas da Reforma Psiquiátrica, pela via de sua passagem direta para a Psiquiatria Comunitária" que influenciou fortemente as práticas em saúde mental no contexto brasileiro, a partir da década de 19702:115.

Este estudo se propõe a analisar aspectos teóricos sobre a saúde mental na atenção básica e sua interface com a psiquiatria comunitária a partir de uma reflexão sobre a produção científica relacionada ao tema.

Para isso, foi realizada uma revisão bibliográfica no período de maio a julho de 2005 , quando foram consultadas as bases de dados (Biblioteca Virtual em Saúde, Bireme, Scielo, Lilacs) e fontes primárias que versam sobre o tema, utilizando as palavraschave: saúde mental, atenção primária, psiquiatria, comunidade, atenção básica. Foram encontradas 142 ocorrências e, destas, utilizadas 20 de acordo com o objetivo do estudo.

\section{SAÚDE MENTAL NA ATENÇÃO BÁSICA E SUA INTERFACE COM A PSIQUIATRIA COMUNITÁRIA}

No fim dos anos de 1950, início dos anos de 1960, surge uma nova tendência em relação aos tratamentos dos transtornos comportamentais, os programas de psiquiatria comunitária. Uma revolução para alguns, um desastre para outros, mas, apesar das críticas positivas e negativas, a pergunta que pairava no ar era como essa mudança de abordagem beneficiaria o paciente, que até então estava restrito aos serviços hospitalares ${ }^{3}$.

$\mathrm{Na}$ Itália e Inglaterra, a reforma psiquiátrica foi realizada no âmbito político e social. 0 respaldo legal dado pelo governo favoreceu o fechamento dos hospitais psiquiátricos. No Canadá foi denominada Revolução Tranqüila. Nos Estados Unidos, a Lei Kennedy em saúde mental fez com que fossem instalados centros comunitários para a área. Na França, foi criada a psiquiatria de setor, com ênfase na programação local das ações de saúde mental, e a reforma espanhola se desenvolveu com a participação e ação dos profissionais da área ${ }^{2,4}$.

A concepção de psiquiatria comunitária não nasceu clara, mesmo para os profissionais que se intitulavam 'da' saúde mental. Havia uma dificuldade relacionada inclusive ao conceito de saúde mental comunitária, em oposição à psiquiatria hospitalar, e ao entendimento do que seria comunidade, em relação aos seus limites.

Vários programas foram desenvolvidos, em especial em Quebec (Canadá) e nos Estados Unidos. Esses programas, por serem pioneiros em relação à abordagem da psiquiatria comunitária, que passaria a ser denominada saúde mental comunitária, receberam incentivos governamentais maciços, e houve uma profusão de pesquisas acadêmicas ao longo dos anos de 1960, diminuindo assim a inquietação em relação aos rumos do modelo de assistência em saúde mental na comunidade ${ }^{5}$.

Durante e após a Segunda Guerra Mundial dois fatos deram um ímpeto ao desenvolvimento da psiquiatria comunitária. 0 primeiro estava relacionado ao número de jovens que retornavam da guerra com sintomas psiquiátricos. A partir desse contexto, as autoridades de saúde dos Estados Unidos constataram que a doença mental era um problema significativo de saúde. A outra questão foi a necessidade de devolver os jovens soldados aos campos de batalha o mais rápido possível, o que possibilitou o desenvolvimento da psicoterapia breve, provocando uma mudança na psicoterapia tradicional ${ }^{4}$.

Após a guerra, os hospitais gerais passaram a aceitar pacientes psiquiátricos, o que viabilizou a permanência dos doentes nas comunidades de origem ${ }^{5}$.

Apesar desses avanços nos anos de 1950, os hospitais psiquiátricos dos Estados Unidos abrigavam cerca de meio milhão de pacientes. As condições precárias de assistência a essa população eram alvo de denúncias e críticas por parte da opinião pública. Em decorrência dessas pressões, o Congresso Americano, em 1955, criou uma Comissão de Enfermidade e Saúde Mental, que, entre os anos de 1955 e 1963, avaliou, discutiu e criou as bases políticas e jurídicas para transformar a assistência psiquiátrica ${ }^{2,4,5}$.

A Comissão apresentou, em 1961, um relatório intitulado Action for Mental Health que recomendava que fossem criadas bases de assistência comunitária, leitos psiquiátricos em hospitais gerais. Os grandes hospitais psiquiátricos deveriam ter seus leitos reduzidos, e outros não poderiam ser criados. 0 espaço de ação da psiquiatria deveria ser expandido e incorporado à comunidade como campo de atuaçãa 2,4,5.

A partir de 1990 ocorreram mudanças em relação às políticas de saúde mental caracterizadas pela reestruturação da assistência psiquiátrica, melhor aplicação dos recursos financeiros e desenvolvimento de serviços integrados à atenção básica. A ênfase era 0 desenvolvimento de dispositivos comunitários visando ao tratamento precoce, contínuo e eficiente na reabilitação e reinserção do usuário da saúde mental ${ }^{6}$.

Para Hersh ${ }^{7}$ não foi surpresa que o desenvolvimento da psiquiatria comunitária ocorresse em um momento de guerra e pobreza, promulgação dos direitos civis, revoluções estudantis e movimentos de liberação e libertação social. 
0 clima sócio-político influenciava nas questões da saúde, não só nas políticas para a área, mas também no agir em saúde, e, nesse contexto reformista, algumas áreas tiveram destaque, em especial as áreas relacionadas ao uso de substâncias psicoativas, suicídio, violência e transtornos comportamentais. Para os progressistas, este pensamento poderia ser perigoso e ferir a individualidade e liberdade de escolha dos cidadãos, já os conservadores acreditavam que o controle social fortalecia o estado e defendiam que a mudança deveria ser realizada paulatinamente ${ }^{7}$.

No Canadá, o pensamento em relação à psiquiatria comunitária após o frenesi causado por sua estruturação foi revisto, e esta passou a ser naturalmente denominada saúde mental comunitária, sendo entendida como um programa de atenção sócio-político que não deveria suplantar os programas de prevenção e promoção em saúde mental. Quanto aos hospitais, estes eram acionados apenas em casos graves e por fim foram desmantelados, rendendo-se enfim à saúde mental comunitária ${ }^{8}$.

A princípio, foram criados serviços intermediários, tais como pequenos hospitais e clínicas para tratamento de transtornos comportamentais menores que reproduziam o modelo do macro hospital. Os pacientes eram identificados como doentes, o prognóstico era sombrio, e o tratamento estava centrado na terapia farmacológica e no médico; com a expansão dos programas de saúde mental comunitária que não pressupunha apenas a implantação de serviços de saúde comunitários, os pacientes e a comunidade começaram a identificar os transtornos como um modo de estar no mundo ${ }^{7-9}$.

A mudança conceitual e prática em relação à atenção destinada à pessoa em sofrimento psíquico rompe com o isolamento em que esse sujeito até então vivia, possibilita que ele planeje projetos de vida, mobiliza seus pares e a comunidade a sua volta; percebe-se que há uma preocupação com o desenvolvimento e manutenção de boas práticas em saúde mental ${ }^{\mathrm{a}}$. 0 caminho em direção à comunidade possibilita a abertura de outras frentes de trabalho e inserção para a saúde mental, em especial nos projetos de prevenção da doença e promoção da saúde.

0 indivíduo deveria ser alcançado, e os programas destinados à população em geral não respeitavam as particularidades e singularidades dos sujeitos. Em resposta, os epidemiologistas entraram em cena e passaram a realizar estudos de coortes para demonstrar que a população era mais importante. Outras pesquisas foram desenvolvidas apontando que a prevenção não deveria ser esquecida e que a psiquiatria comunitária inspirada nos programas de saúde pública deveria ir ao encontro da atenção primária8.

Além de focar a saúde mental, esses programas deveriam desenvolver ações sociais e promover a qualidade de vida da comunidade. Os profissionais de saúde mental deveriam ser encorajados a promover reformas sociais a partir de sua prática, de seu contato com a comunidade, ajudando a mesma a se organizar em busca de melhores condições de tratamento. Há incorporação de novos profissionais às equipes, consolidando a idéia do ser biopsicossocial que merece ser acompanhado de diferentes formas e por diversos saberes, incluindo aqui a sua demanda. Em última análise, a finalidade era diminuir a vulnerabilidade dos indivíduos a certos transtornos comportamentais menores e acompanhar os demais casos em suas particularidades ${ }^{6-8}$.

Algumas características foram atribuídas à prática da saúde mental na atenção básica, entre elas: estar associada às demais ações da atenção básica; assegurar o bem estar da comunidade e do indivíduo; privilegiar as ações preventivas, individuais e coletivas; alocar os programas de saúde mental em diferentes serviços de atenção básica, formando uma rede de suporte e cuidados; realizar ações diretas e indiretas; utilizar novas estratégias de abordagem em saúde; ter governabilidade; agregar profissionais com diferentes formações, implicar a comunidade e, por fim, considerar as características da comunidade ${ }^{10}$.

Após a transposição da psiquiatria comunitária para a atenção primária, algumas fragilidades foram identificadas, entre elas: a fragmentação dos programas com o tempo; a imprecisão dos limites da saúde mental na atenção básica; e a dificuldade da integração dos saberes dos profissionais envolvidos. E foi levantada, em dado momento, a questão de se a psiquiatria comunitária/saúde mental comunitária não poderia estar perdendo seu foco de atuação $0^{6-10}$.

No Brasil, apenas na década de 1970 houve a incorporação dessas mudanças ocorridas nos demais países aqui apresentados, nesse momento a designação biopsicossocial passou a ser a característica das práticas da Saúde Mental Comunitária ${ }^{5}$.

Apesar da mudança no enfoque da atenção psiquiátrica, a psiquiatria comunitária apresentava a preocupação com 0 binômio saúde-doença com ênfase na doença. Sendo a psiquiatria comunitária uma disciplina médica que incorpora conceitos oriundos da saúde pública e postula a organização dos serviços de assistência psiquiátrica e social, o que ora observamos em nosso meio é que, apesar de estar inserida na comunidade, ela ainda trabalha com a proposta de encaminhamentos para o hospital psiquiátrico e nem sempre desenvolve as ações propostas ${ }^{2,5}$.

Cabe ressaltar que ainda temos, em nosso meio, a presença marcante do hospital psiquiátrico, que vem sendo utilizado pelos serviços substitutivos como ponto de apoio quando estes esgotam sua capacidade de argumentação com a loucura do outro, quer seja por excesso de demanda ou pelo que se convencionou chamar de fim das possibilidades terapêuticas, o que acreditamos ser reflexo do aumento da procura pelos serviços de saúde mental substitutivos e do esgotamento das equipes, entre outros pontos que devem ser observados e pesquisados com atenção.

A psiquiatria comunitária considera, além da participação da comunidade, ações integradas e localizadas em um contexto específico na comunidade, e que essas ações devem ter níveis de análises e eixos de gestão em saúde que diferem em suas propostas de intervenção. Uma aproximação possível é diferenciar a psiquiatria que se faz "para" a comunidade, "na" comunidade e "com" a comunidade ".

No primeiro caso, "para" a comunidade se refere às iniciativas dos serviços de saúde mental que planificam as ações, mas considerando as conseqüências que tais ações possam ter sobre a comunidade, em um sentido social cotidiano. $\mathrm{Na}$ psiquiatria "na" comunidade, as iniciativas atravessam o trabalho da saúde mental e buscam ser mais acessíveis à população, como, por exemplo, na atenção primária à saúde ${ }^{11}$. 
Essa forma de pensar a psiquiatria considera que a proximidade física com 0 contexto do paciente facilita 0 acesso da equipe no sentido de tornar-se familiar. No terceiro caso, em que temos a psiquiatria "com" a comunidade, teremos ações que são motivadas pelos problemas que a comunidade identifica. Há uma resposta efetiva a um problema, por meio de uma relação direta ${ }^{10,11}$.

Em relação à primeira característica, podemos considerar que esse tipo de aproximação requer uma visão sociológica da medicina, em que os problemas de saúde são definidos como perda de oportunidades pelos sujeitos no meio em que vivem decorrentes da situação de doença. 0 diagnóstico desse sujeito adquire maior importância do que as oportunidades da vida em sociedade. São as perdas percebidas pelo sujeito durante a doença que devem ser consideradas durante o planejamento da assistência ${ }^{11}$.

Os problemas que a comunidade percebe como relevantes quase sempre superam a capacidade de resolução das equipes de saúde, pois requerem, por parte destas, oferecer recursos que realmente dispõem, e não encaminhamentos que não respondam aos seus anseios.

0 profissional de saúde sob essa perspectiva mobiliza as capacidades e recursos do paciente e da comunidade, enfatizando o protagonismo do sujeito para resolver seus problemas.

Existem certas características que devem ser comuns aos serviços comunitários, entre elas: proximidade; individualidade; clareza em relação aos direitos e deveres dos prestadores e beneficiários das ações de saúde do princípio ao término da atenção e da relação terapêutica reabilitadora; continuidade da atenção e respeito aos direitos do usuário, independente do estado de saúde em que se encontre ${ }^{12}$.

Os profissionais de saúde envolvidos devem assumir que os resultados da proposta desse tipo de programa são coletivos, e não individuais. Eles devem aceitar que os resultados serão conhecidos e reconhecidos por meio de sua eficiência e que isso requer retroalimentação do usuário ${ }^{10}$. Uma relação em que os sujeitos em sofrimento mental ditam regras implica a perda do poder dos profissionais de saúde historicamente instituído, e nem sempre essa mudança acontece sem embates ou resistências.

Em todas as experiências em psiquiatria comunitária, existem consensos do que seja o melhor para o paciente. Por exemplo, é na comunidade que o sujeito, apesar de doente, pode elaborar seus projetos de vida, partindo do princípio de que a comunidade é o seu ambiente familiar e que a vida nesse ambiente é um constituinte indispensável para o processo de saúde do sujeito. Esta afirmação se fundamenta na compreensão de que o sujeito doente tem mais probabilidades de alcançar sua normalidade se viver e for tratado em um ambiente "[...] o mais parecido possível a seu lugar de origem [...]"13:132.

Este ambiente oferece ao paciente as condições fundamentais para sua recuperação, que são, entre outras: estar rodeado de estímulos positivos e saudáveis segundo suas crenças; ser tratado com compreensão e flexibilidade sem perder a individualidade. ${ }^{13}$ Por outro lado, neste tipo de situação, aumenta a probabilidade do diagnóstico e tratamento precoce dos transtornos comportamentais, dado o controle social importante que pode ter uma população informada ${ }^{12}$.
É no trabalho das equipes de saúde, "com e na" comunidade, que se produzirão inevitavelmente as melhores oportunidades de controle e intervenção por parte de terceiros sobre o que está ocorrendo com o paciente ${ }^{11}$.

Deste ponto de vista, a psiquiatria comunitária se expressa em uma ação de cuidados em psiquiatria e saúde mental, dirigida à comunidade (geograficamente limitada), bem definida em todas as suas características sociais e demográficas. Sua ênfase está na vigilância desta comunidade (detecção e controle precoce de fatores de risco e de evidências de ordem social que possam causar sofrimento mental) e no acompanhamento dos pacientes sabidamente em sofrimento, estando ou não em tratamento medicamentoso ${ }^{11}$.

A mudança no contexto dos serviços de saúde mental, tradicionalmente voltado para o tratamento individual e organizado, segundo um modelo médico e hospitalocêntrico, para o desenvolvimento de ações na comunidade, tem oferecido contribuições históricas tanto no que concerne a trabalhos com grupos e comunidades como a aportes teóricos relacionando a influência dos fenômenos sociais nos transtornos comportamentais.

No Brasil, algumas iniciativas de atenção psiquiátrica comunitária têm apresentado bons resultados, entre elas a criação de condições para assistência aos pacientes psiquiátricos na comunidade com 0 suporte do hospital geral ou equipes de saúde mental ligadas ao PSF, para intervenções em crise e acompanhamento domiciliar, partindo do princípio do compartilhamento de responsabilidades no cuidado e no acolhimento ${ }^{14,15}$.

Apesar das dificuldades relacionadas aos recursos financeiros para as ações, atualmente existe a compreensão de que é possível desenvolver ações de saúde mental na comunidade, e que esta deve ser vista como um dos espaços de atuação dessa especialidade. A realidade das equipes de atenção básica demonstra um quadro preocupante e ao mesmo tempo estimulante: $56 \%$ das equipes de saúde da família referiram realizar "alguma ação de saúde mental" ${ }^{16}$.

A questão é como essas equipes estão lidando com essa realidade. As equipes estão prontas para lidar com essa demanda? As equipes matriciais de saúde mental estão trabalhando efetivamente para assegurar que esses usuários sejam atendidos em sua comunidade, proporcionando um atendimento de qualidade e capacitando os profissionais da atenção básica para realizar tal atividade com segurança? Há integralidade das ações?

0 Ministério da Saúde editou a Norma Operacional da Assistência à Saúde-01/2001, que, entre outros pontos, orienta a organização do Plano Diretor de Regionalização, determinando que o cidadão deva ter acesso, o mais próximo possível de sua residência, a um conjunto de ações e serviços vinculados às responsabilidades mínimas, dentre as quais o tratamento dos transtornos comportamentais e psicossociais mais freqüentes ${ }^{14-16}$.

A aprovação da Lei 10.216, de 06 de abril de 2001, dispõe sobre a proteção e os direitos das pessoas portadoras de transtornos mentais e direciona o modelo assistencial em saúde mental. Na sua definição dos direitos da pessoa portadora de transtorno mental, diz, no item IX do parágrafo único do artigo $2^{\circ}$, que a pessoa assistida deve ser tratada, preferencialmente, em serviços comunitários de saúde mental ${ }^{14,15}$. 
As ações de saúde mental na atenção básica devem estar fundamentadas nos princípios do Sistema Único de Saúde e nos princípios da Reforma Psiquiátrica, trabalhando em redes, com base territorial e atuação transversal com outras políticas específicas. A proposta de trabalho do Ministério da Saúde ${ }^{14-}$ ${ }^{16}$ para a inclusão das ações de saúde mental na atenção básica, direciona e traça um plano de trabalho conjunto que utiliza equipes matriciais de saúde mental na organização das ações de saúde mental na atenção básica.

A inclusão de indicadores de saúde mental no Sistema de Informações da Atenção Básica (SIAB), entre eles o número de pessoas identificadas com transtornos psiquiátricos graves e o percentual de pessoas com transtorno mental egressas de internação psiquiátrica acompanhadas pela rede básica por faixa etária e sexo, são avanços para a área. As diretrizes do Ministério da Saúde em relação aos recursos humanos em saúde mental especificam o termo equipe mínima, embora não conste em seus documentos o termo equipe multidisciplinar; este ficaria por parte das equipes e de sua constituição, como um requisito da proposta terapêutica e dos recursos financeiros dos municípios responsáveis pela implantação e continuidade do serviço ${ }^{14,15}$.

0 modelo assistencial em saúde mental permanece em transição, refletindo, em alguns serviços, as graves distorções do modelo asilar, embora haja uma compreensão de que atualmente as políticas públicas para a área são necessariamente intersetoriais, com interface com áreas como a assistência social, os direitos humanos, a justiça, o trabalho e a habitaçãa $0^{14-17}$.

Em relação à inclusão das ações de saúde mental na atenção básica, a compreensão de que elas são indissociáveis da atenção primária em saúde, a capacitação das equipes do Programa de Saúde da Família para o manejo e tratamento dos transtornos comportamentais e a inclusão e 0 acompanhamento das ações de saúde mental na rede básica já são realidade em alguns municípios ${ }^{16,18}$.

\section{Referências}

1. Donnangelo MCF.Saúde e sociedade. São Paulo (SP): Duas Cidades; 1997.

2. Costa-Rosa A. Saúde mental comunitária: análise dialética das práticas alternativas. [dissertação mestrado]. São Paulo (SP): Instituto de Psicologia/USP; 1987.

3. Davidson L. Deinstitutionalitation and community based psychiatry: some aspects from literature. Med Arh 1999 Sept; 53(3): 135-38.

4. Gondim DSM. Análise da implantação de um serviço de emergência psiquiátrica no município de campos: inovação ou reprodução do modelo assistencial? [dissertação mestrado]. Rio de Janeiro [RJ]: Escola Nacional de Saúde Pública/FIOCRUZ; 2001.

5. Birman J, Costa JF. Organização de instituições para uma psiquiatria comunitária. In: Amarante P, organizador. Psiquiatria social e reforma psiquiátrica. Rio de Janeiro (RJ): FIOCRUZ; 1994.
Existe um aparato juríico, ético e científico que impulsiona as ações de saúde mental na comunidade. 0 campo para a discussão e reflexão sobre o tema está aberto, a reforma se mantém. A política para a área está em transformação no campo dos saberes, das práticas, da cultura e no campo jurídico. É um movimento que merece ser acompanhado de perto diante de seu potencial dinâmico e criativo e dos benefícios que proporcionam à população.

\section{CONSIDERAÇÕES FINAIS}

0 resgate do doente mental para o convívio social implica endereçar à comunidade a pluralidade de aspectos presentes no convivio da pessoa em sofrimento psíquico na vida social. 0 convívio com a desigualdade social, o ambiente de violência e desemprego e 0 transtorno comportamental fazem parte do cotidiano da comunidade.

As transformações dos saberes e práticas na clínica psiquiátrica não podem ser esquecidas nesse momento de efervescência, em que estamos descobrindo que a relação entre o normal e o patológico simplesmente nos diz que não existe um jeito normal de ser, é o outro jeito de ser e estar no mundo que deve ser compreendido.

A ação conjunta entre a saúde mental e a atenção básica é uma realidade a qual, diante do contingente de pessoas que sofrem com o transtorno comportamental e hoje são atendidas pelas equipes de saúde da família, se tornou necessária.

No enfrentamento desses desafios, algumas questões devem ser priorizadas, tais como qualificar 0 atendimento, por meio da capacitação, do suporte matricial e da incorporação dos casos de transtornos psíquicos graves à assistência no território.

Os desafios estão presentes e não podem ser ignorados. 0 sofrimento mental está cada vez mais presente na sociedade e deve ser superado, quer seja avançando no conhecimento em relação às diretrizes da política de saúde mental ou aproximando-nos das contingências do processo de adoecimento e contribuindo para consolidar a saúde mental no âmbito da atenção básica.

6. Coplan B. Principles of psychiatry community. Community Mental Health J 2000 Sept; 36 (1): 7-24.

7. Hersh C. The discontent explosion in mental health. Am Psychol 1968 Jun; 2(3): 497-506.

8. Blom BLK. Community mental health: a general introduction. Monterey (CA): Brooks Cole; 1975.

9. Cohen NI. Training in community psychiatric: new opportunities. Psychiatr J 1998; 69 (2): 107-09.

10. Inpes G. Psychiatrie et santé mentale: 2005-2008. Orientations au service: politique publique de psychiatric et de santé mentale. Paris (FR); 2007.

11. World Health Organization-WHO. Nations for mental health supporting governments and policy-makers. [documento online] 1998 Sept [citado 25 jun 2003] : 228 Disponível em: www.who.int/mental_health. 
12. Freitas FFP. Subsídios para mudanças do modelo de assistência psiquiátrica. Cad Saude Publica 1998 jan/mar; 14(1): 93-106.

13. Jacobson $L$. The transitions from mental hospital based to community psychiatry. Med Arh 1999; 53 (3):131-33.

14. Saúde mental e a atenção básica. Dynamis: rev tecno-cient 2004 abr/jun; 12 (47 p.2).

15. Saúde mental na atenção básica. Cad IPUB 2007 mar/abr; 13 (24):

16. Lancetti $A$, et al. Saúde loucura. $2^{\mathrm{a}}$ ed. São Paulo (SP): Hucitec; 1991.

17. Amarante $P$, organizador. Archivos de saúde mental e atenção psicossocial. Rio de janeiro (RJ): NAU Ed; 2003.

18. Organização Panamericana de Saúde-OPS/Organização Mundial de Saúde-OMS. Declaração de Caracas. Conferência Regional sobre reestruturação da atenção psiquiátrica no contexto dos sistemas locais de saúde; 1992. p. 5.

\section{Nota}

Diante das orientações das políticas de saúde mental, a autora tem pesquisado e trabalhado a necessidade de desenvolver e aprimorar as práticas profissionais dos enfermeiros psiquiátricos, favorecendo assim o desenvolvimento constante da qualidade de suas ações por meio da construção do conceito de boas práticas em saúde mental. Esse trabalho compreende a elaboração de instrumentos que possam avaliar as intervenções realizadas pelo enfermeiro junto ao usuário, família e comunidade, considerando a diversidade de ações desenvolvidas pelo enfermeiro e as diversas referências teóricas que amparam essas práticas. Considera também a evolução das estratégias terapêuticas, seus benefícios, riscos, eficiência e custos, favorecendo o desenvolvimento e formalização do conceito. 0 mesmo vem sendo desenvolvido por meio de diferentes projetos de pesquisa coordenados pela autora. 